\title{
BIHARMONIC CAPACITY AND THE STABILITY OF MINIMAL LAGRANGIAN SUBMANIFOLDS
}

\author{
BENNETT PALMER
}

(Received November 5, 2001, revised November 18, 2002)

\begin{abstract}
We study the eigenvalues of the biharmonic operators and the buckling eigenvalue on complete, open Riemannian manifolds. We show that the first eigenvalue of the biharmonic operator on a complete, parabolic Riemannian manifold is zero. We give a generalization of the buckling eigenvalue and give applications to studying the stability of minimal Lagrangian submanifolds in Kähler manifolds.
\end{abstract}

1. Introduction. This paper is concerned with eigenvalues of the biharmonic operators and the buckling eigenvalue for complete Riemannian manifolds. We are mostly concerned with relating bounds for these eigenvalues to the behavior of the ends of the manifold. Let $M$ be a complete Riemannian manifold. $M$ is called parabolic if every non-positive subharmonic function on $M$ reduces to a constant. By an $e$ d $\mathcal{E}$ of $M$ we mean an unbounded connected component of the complement of a compact subset. An end $\mathcal{E}$ will be called parabolic if there exists a complete parabolic Riemannian manifold whose only end is $\mathcal{E}$. If $M$ is a surface these concepts are conformally invariant.

Let $\Omega \subset \subset M$ be a relatively compact subdomain with smooth boundary. Let $P>0$ be a smooth function on $M$ and consider the eigenvalue problem

$$
\begin{gathered}
\Delta^{2} u-\Lambda P u=0 \quad \text { in } \Omega, \\
u=\partial_{n} u=0 \quad \text { on } \partial \Omega,
\end{gathered}
$$

where $\Delta^{2}=\Delta \Delta$ denotes the biharmonic operator. Denote the eigenvalues by $\Lambda_{1} \leq \Lambda_{2} \leq$ $\Lambda_{3} \leq \cdots, \Lambda_{j}=\Lambda_{j}(P, \Omega)$. Let

$$
\Lambda_{1}(P, M):=\inf _{\Omega \subset \subset M} \Lambda_{1}(P, \Omega) .
$$

THEOREM 1.1. If $M$ is a complete, open, parabolic Riemannian manifold, then $\Lambda_{1}(P, M)=0$.

We recall a well-known result of Fischer-Colbrie and Schoen which states that any complete, minimal surface in a three dimensional manifold with uniformly positive scalar curvature is unstable in the sense that there are compactly supported variations of the surface which decrease its area. Oh [4] introduced the concept of Hamiltonian stability for minimal Lagrangian submanifolds in a Kähler manifold. In view of the similarities between minimal

2000 Mathematics Subject Classification. Primary 53A10; Secondary 35P15, 58E12.

Key words and phrases. Minimal Lagrangian submanifold, buckling eigenvalue. 
surfaces in 3-manifolds and minimal Lagrangian surfaces in Kähler manifold of real dimension 4 , we are lead to the following conjecture:

Let $f: \Sigma^{2} \rightarrow X^{4}$ be a minimal, Lagrangian immersion of an open surface into a Kähler 4-manifold whose Ricci curvature has a positive lower bound. Assume that the metric induced on $\Sigma$ is complete. Then the immersion is not Hamiltonian stable.

In Section 5 we give results in this direction. For a subdomain $\Omega$ of a Riemannian manifold, we let $C_{c}^{\infty}(\Omega)$ denote the space of smooth functions with compact support in $\Omega$. We introduce a natural generalization of buckling eigenvalue

$$
\beta_{1}(\Omega):=\inf _{C_{c}^{\infty}(\Omega)} \frac{\int(\Delta u)^{2}}{\int|\nabla u|^{2}},
$$

which we use to study the stability of minimal, Lagrangian submanifolds in Kähler manifolds. We show:

A complete minimal Lagrangian submanifold with at least two nonparabolic ends in a Kähler manifold with uniformly positive Ricci curvature is not Hamiltonian stable.

In particular, we verify the conjecture given above in the case where the surface has at least two nonparobolic ends. We also provide a large supply of examples to which this result applies.

The author would like to express his appreciation to Dr. John Parker for a helpful conversation concerning the examples at the end of Chapter 5.

In the final section, we use an idea of Payne [7] to give an upper bound for the buckling eigenvalue of a relatively compact, convex domain in a Riemannian manifold in terms of the first Dirichlet eigenvalue of the Laplacian and a lower bound for the Ricci curvature.

2. Capacities. Let $\left\{\Omega_{j}\right\}_{j=0,1,2, \ldots}$ be an exhaustion of a Riemannian manifold $M$ by relatively compact subdomains, and let $A_{j}:=\Omega_{j}-\bar{\Omega}_{0} ; j=1,2, \ldots$ Let $\omega_{j}$ denote the harmonic measure of $\partial \Omega_{j}$ with respect to $A_{j}$, satisfying

$$
\begin{aligned}
\Delta \omega_{j}=0 & \text { in } A_{j}, \\
\omega_{j}=0 & \text { on } \partial \Omega_{0}, \\
\omega_{j}=1 & \text { on } \partial \Omega_{j} .
\end{aligned}
$$

The (harmonic) capacity of $A_{j}$ is defined to be

$$
\frac{1}{\mu_{j}}:=\int_{A_{j}}\left|\nabla \omega_{j}\right|^{2} .
$$

It is well-known that $M$ is parabolic if and only if

$$
\lim _{j \rightarrow \infty} \frac{1}{\mu_{j}}=0 .
$$


Let $v_{j}$ be the biharmonic function defined by

$$
\begin{aligned}
\Delta^{2} v_{j}=0 \quad \text { in } A_{j}, \\
\partial_{n} v_{j}=0 \quad \text { on } \partial A_{j}, \\
v_{j}=\omega_{j} \quad \text { on } \partial A_{j} .
\end{aligned}
$$

In analogy with the above we define the biharmonic capacity of $A_{j}$ by

$$
\frac{1}{v_{j}}:=\int_{A_{j}}\left(\Delta v_{j}\right)^{2} .
$$

For any region $\Omega \subset M$, define $L_{H}^{2}(\Omega)=\left\{h \in L^{2}(\Omega) \mid \Delta h=0\right\}$. Note that by Weyl's lemma $L_{H}^{2}(\Omega)$ is a closed subspace of $L^{2}(\Omega)$.

LEMMA 2.1.

(i)

$$
\begin{aligned}
1 / \mu_{j}=\sup _{L_{H}^{2}\left(A_{j}\right)} \frac{\left(\oint_{\partial \Omega_{j}} * d h\right)^{2}}{\int_{A_{j}}|\nabla h|^{2}} . \\
1 / \nu_{j}=\sup _{L_{H}^{2}\left(A_{j}\right)} \frac{\left(\oint_{\partial \Omega_{j}} * d h\right)^{2}}{\int_{A_{j}} h^{2}} .
\end{aligned}
$$

Proof. Let $h \in L_{H}^{2}\left(A_{j}\right)$. Then

$\left(\oint_{\partial \Omega_{j}} * d h\right)^{2}=\left(\oint_{\partial A_{j}} \omega_{j} * d h\right)^{2}=\left(\int_{A_{j}}\left\langle\nabla \omega_{j}, \nabla h\right\rangle\right)^{2} \leq\left(\int_{A_{j}}\left|\nabla \omega_{j}\right|^{2}\right)\left(\int_{A_{j}}|\nabla h|^{2}\right)$ with equality if $h=\omega_{j}$. To prove (ii) note that

$$
\begin{aligned}
\left(\oint_{\partial \Omega_{j}} * d h\right)^{2} & =\left(\oint_{\partial A_{j}} v_{j} * d h\right)^{2}=\left(\oint_{\partial A_{j}} h * d v_{j}+\int_{A_{j}}\left(v_{j} \Delta h-h \Delta v_{j}\right)\right)^{2} \\
& =\left(\int_{A_{j}} h \Delta v_{j}\right)^{2} \leq\left(\int_{A_{j}} h^{2}\right)\left(\int_{A_{j}}\left(\Delta v_{j}\right)^{2}\right)
\end{aligned}
$$

with equality if $h=\Delta v_{j}$.

COROLlaRY 2.1. The sequence $\left\{1 / v_{j}\right\}_{j=1,2, \ldots}$ decreases monotonically.

Proof. Let $j^{\prime}>j>0$ and $h_{j^{\prime}}:=\Delta v_{j^{\prime}}$. Then

$$
\frac{1}{v_{j}} \geq \frac{\left(\oint_{\partial \Omega_{j}} * d h_{j^{\prime}}\right)^{2}}{\int_{A_{j}} h_{j^{\prime}}^{2}}>\frac{\left(\oint_{\partial \Omega_{j}^{\prime}} * d h_{j^{\prime}}\right)^{2}}{\int_{A_{j^{\prime}}} h_{j^{\prime}}^{2}}=\frac{1}{v_{j^{\prime}}} .
$$


Let $B_{r}$ denote the geodesic ball about a fixed $p \in M$. For $0<r<R$ let $A_{r, R}:=$ $B_{R}-\bar{B}_{r}$. Let $\omega_{r, R}$ denote the harmonic measure of $\partial B_{R}$ with respect to $A_{r, R}$.

LEMMA 2.2. Let $0<r<R$ and $0 \leq a<b \leq 1$. Let $(1 / \mu)\left(\left\{a \leq \omega_{r, R} \leq b\right\}\right)$ denote the harmonic capacity of $\left\{a \leq \omega_{r, R} \leq b\right\}$, i.e.,

$$
(1 / \mu)\left(\left\{a \leq \omega_{r, R} \leq b\right\}\right):=\int_{\left\{a \leq \omega_{r, R} \leq b\right\}}|\nabla \omega|^{2},
$$

where $\omega$ is the harmonic function with constant value 0 on $\left\{\omega_{r, R}=a\right\}$ and constant value 1 on $\left\{\omega_{r, R}=b\right\}$. Then

$$
(1 / \mu)\left(\left\{a \leq \omega_{r, R} \leq b\right\}\right) \leq \frac{1}{(b-a)^{2}} \frac{1}{\mu_{r, R}} .
$$

ProOF. Simply note that

$$
\omega=\frac{\omega_{r, R}-a}{b-a}
$$

Therefore

$$
\int_{\left\{a \leq \omega_{r, R} \leq b\right\}}|\nabla \omega|^{2} \leq \frac{1}{(b-a)^{2}} \frac{1}{\mu_{r, R}} .
$$

\section{Bounds for the biharmonic capacity.}

Proposition 3.1. If $M$ is a complete parabolic manifold, then

$$
\lim _{R \rightarrow \infty} 1 / v_{r, R}=0
$$

Proof. Without loss of generality we may assume $r=1$. Let $h \in L_{H}^{2}\left(A_{1, R}\right)$. Then for any $\zeta \in C_{c}^{\infty}\left(A_{1, R}\right)$

$$
0=\oint_{\partial A_{1, R}} \zeta^{2} h * d h=\int_{A_{1, R}}\left(\zeta^{2}|\nabla h|^{2}+2 \zeta h\langle\nabla \zeta, \nabla h\rangle\right)
$$

Therefore

$$
\int_{A_{1, R}} \zeta^{2}|\nabla h|^{2} \leq 2 \int_{A_{1, R}}|\zeta h\langle\nabla \zeta, \nabla h\rangle| \leq 2\left(\int_{A_{1, R}} \zeta^{2}|\nabla h|^{2}\right)^{1 / 2}\left(\int_{A_{1, R}} h^{2}|\nabla \zeta|^{2}\right)^{1 / 2}
$$

and hence

$$
\int_{A_{1, R}} \zeta^{2}|\nabla h|^{2} \leq 4 \int_{A_{1, R}} h^{2}|\nabla \zeta|^{2}
$$

Now consider the region $\left\{1 / 2 \leq \omega_{1, R / 2}\right\} \subset A_{1, R / 2}$. Let

$$
\rho:=\operatorname{distance}\left(\partial B_{1},\left\{1 / 2 \leq \omega_{1, R / 2}\right\}\right) .
$$

Since $M$ is assumed to be parabolic, the family of functions $\left\{\omega_{1, R / 2}\right\}$ converge to zero uniformly on compact subsets of $M-B_{1}$ and hence

$$
\lim _{R \rightarrow \infty} \rho=\infty \text {. }
$$


One sees that there exists a function $\zeta_{R} \in C_{0}^{\infty}\left(A_{1, R}\right)$ such that $\zeta_{R} \equiv 1$ on $\left\{1 / 2 \leq \omega_{1, R / 2}\right\}$ and

$$
\left|\nabla \zeta_{R}\right|^{2} \leq c\left(1 / R^{2}+1 / \rho^{2}\right),
$$

where $c$ is a constant independent of $R$. It therefore follows from (6) that

$$
4 c\left(1 / R^{2}+1 / \rho^{2}\right) \int_{A_{1, R}} h^{2} \geq \int_{\left\{1 / 2 \leq \omega_{1, R / 2}\right\}}|\nabla h|^{2} .
$$

Therefore, using (i) of Lemma 2.1 and Lemma 2.2 we obtain

$$
\begin{aligned}
16 c\left(1 / R^{2}+1 / \rho^{2}\right)\left(1 / \mu_{1, R / 2}\right) & \geq 4 c\left(1 / R^{2}+1 / \rho^{2}\right) \frac{\left(\oint_{\partial B_{R}} * d h\right)^{2}}{\int_{\left\{1 / 2 \leq \omega_{1, R / 2}\right\}}|\nabla h|^{2}} \\
& \geq \frac{\left(\oint_{\partial B_{R}} * d h\right)^{2}}{\int_{A_{1, R}} h^{2}},
\end{aligned}
$$

and hence using (ii) of Lemma 2.1

$$
16 c\left(1 / R^{2}+1 / \rho^{2}\right)\left(1 / \mu_{1, R / 2}\right) \geq \frac{1}{v_{1, R}}
$$

and the result follows.

Proof of TheOREM 1.1. The first eigenvalue $\Lambda_{1}(P, \Omega)$ appearing in (1) is characterized by

$$
\Lambda_{1}=\inf _{C_{c}^{\infty}(\Omega)} \frac{\int_{\Omega}(\Delta f)^{2}}{\int_{\Omega} P f^{2}} .
$$

Define a function $u$ on $B_{R}$ by

$$
u(x):= \begin{cases}1 & x \in \bar{B}_{1}, \\ 1-v_{1, R} & x \in A_{1, R},\end{cases}
$$

Then $u$ lies in the Sobolev space $W_{0}^{2,2}\left(B_{R}\right)$ of functions which have square integrable distributional derivatives of orders less than or equal to two and which vanish to first order on the boundary. The Laplacian of $u$ is given by

$$
\Delta u(x):= \begin{cases}0 & x \in B_{1}, \\ -\Delta v_{1, R} & x \in A_{1, R} .\end{cases}
$$

By taking $f=u$, we obtain

$$
\Lambda_{1}\left(B_{R}\right) \int_{B_{1}} P \leq \frac{1}{v_{1, R}} .
$$

The result then follows from Proposition 3.1. 
4. Buckling eigenvalues. Let $M$ be a complete Riemannian manifold and let $\Omega$ be a relatively compact subdomain with smooth immersed boundary. The buckling eigenvalue of $\Omega$ is defined by

$$
\beta_{1}(\Omega):=\inf _{C_{c}^{\infty}(\Omega)} \frac{\int(\Delta u)^{2}}{\int|\nabla u|^{2}} .
$$

There exists a smooth function $u$ on $\Omega$ satifying

$$
\begin{aligned}
\Delta^{2} u+\beta_{1} \Delta u=0 & \text { in } \Omega, \\
\partial_{n} u=0 & \text { on } \partial \Omega, \\
u=0 & \text { on } \partial \Omega .
\end{aligned}
$$

We define the generalized buckling eigenvalue $\alpha_{1}$ as follows. Let $\partial \Omega=\gamma_{1} \cup \cdots \cup \gamma_{n}$ be the decomposition of $\partial \Omega$ into disjoint components. Let $w_{i}$ denote the solution of

$$
\begin{aligned}
\Delta^{2} w_{i} & =0 & & \text { in } \Omega, \\
\partial_{n} w_{i} & =0 & & \text { on } \partial \Omega, \\
w_{i} & =\delta_{i, j} & & \text { on } \gamma_{j},
\end{aligned}
$$

and let $W:=\operatorname{span}\left\{w_{1}, \ldots, w_{n}\right\}$. Define

$$
\alpha_{1}=\alpha_{1}(\Omega):=\inf _{u \in C_{c}^{\infty}(\Omega) \oplus W} \frac{\int(\Delta u)^{2}}{\int|\nabla u|^{2}} .
$$

It can be shown by direct methods that the infimum is attained by a $C^{4}(\Omega)$ function $u$ satisfying

$$
\begin{gathered}
\Delta^{2} w+\alpha_{1} \Delta w=0 \quad \text { in } \Omega \\
\partial_{n} w \equiv 0 \quad \text { on } \partial \Omega \quad w \equiv c_{j} \quad \text { on } \gamma_{j},
\end{gathered}
$$

where $c_{j}$ are constants.

Note the trivial inequality $\alpha_{1} \leq \beta_{1}$ and that the two agree if $\partial \Omega$ is connected.

Proposition 4.1. Let $\Omega \subset \subset \Omega^{\prime} \subset \subset M$. Assume that $\partial \Omega^{\prime}$ is connected and that $\Omega=\Omega^{\prime}-\left(\bar{D}_{1} \cup \cdots \cup \bar{D}_{n}\right)$, where each $D_{j}$ is a subdomain with connected boundary. Then

$$
\alpha_{1}(\Omega) \geq \beta_{1}\left(\Omega^{\prime}\right) \geq \lambda_{1}\left(\Omega^{\prime}\right) .
$$

Proof. Let $u$ be a competing function in (10). We assume $u \equiv 0$ on $\partial \Omega^{\prime}$ by subtracting an apropriate constant from $u$ if necessary. Let $c_{j}, j=1, \ldots, n$ denote the constant value $c_{j}=\left.u\right|_{\partial D_{j}}$ and define

$$
u^{\prime}(x):= \begin{cases}u & x \in \Omega, \\ c_{j} & x \in \bar{D}_{j} .\end{cases}
$$


Then $u^{\prime} \in W_{0}^{2,2}\left(\Omega^{\prime}\right)$ and therefore

$$
\beta_{1}\left(\Omega^{\prime}\right) \leq \frac{\int_{\Omega^{\prime}}\left(\Delta u^{\prime}\right)^{2}}{\int_{\Omega^{\prime}}\left|\nabla u^{\prime}\right|^{2}}=\frac{\int_{\Omega}(\Delta u)^{2}}{\int_{\Omega}|\nabla u|^{2}} .
$$

This proves the first inequality in (12) by taking the infimum of the last quotient on the right. The second inequality in (12) was proved in [5].

In two dimensions we can relate $\alpha_{1}$ to another widely studied eigenvalue problem.

THEOREM 4.1. Let $\left(M^{2}, \partial M\right)$ be a compact surface with smooth boundary. Denote the Gaussian curvature by K. Let $\sigma_{1}$ denote the first eigenvalue of the problem:

$$
\Delta u-K u+\sigma u=0, \quad \text { in } M \quad u \equiv 0 \quad \text { on } \partial M .
$$

Then $\alpha_{1} \geq \sigma_{1}$ holds.

PROOF. Let $w$ be the function appearing in (11). By the Lichnerowicz formula, we have

$$
\Delta \frac{1}{2}|\nabla w|^{2}=|\nabla \nabla w|^{2}+K(\nabla w, \nabla w)+\langle\nabla w, \nabla \Delta w\rangle .
$$

Expanding out the left hand side gives

$$
|\nabla w| \Delta|\nabla w|+|\nabla| \nabla w||^{2}=|\nabla \nabla w|^{2}+K(\nabla w, \nabla w)+\langle\nabla w, \nabla \Delta w\rangle .
$$

Combining this with Kato's inequality,

$$
|\nabla| \nabla w|| \leq|\nabla \nabla w|,
$$

yields the distributional inequality

$$
|\nabla w| \Delta|\nabla w|-K|\nabla w|^{2} \leq\langle\nabla w, \nabla \Delta w\rangle .
$$

Note that $|\nabla w| \equiv 0$ holds on the boundary. Integrating and using the variational characterization of $\sigma_{1}$ then gives

$$
\begin{aligned}
\sigma_{1} \int|\nabla w|^{2} & \leq-\int\left(|\nabla w| \Delta|\nabla w|-K|\nabla w|^{2}\right) \\
& \leq-\int\langle\nabla w, \nabla \Delta w\rangle=\int(\Delta w)^{2}=\alpha_{1} \int|\nabla w|^{2},
\end{aligned}
$$

from which the result follows.

Let $M$ be a complete open Riemannian manifold. We define

$$
\alpha_{1}(M):=\inf _{\Omega \subset \subset M} \alpha_{1}(\Omega) .
$$

THEOREM 4.2. Let $M$ be a complete, open Riemannian manifold with at least two nonparabolic ends. Then $\alpha_{1}(M)=0$. 
REMARK. Let $M$ denote the Poincare disc equipped with the metric of constant curvature -1 . Clearly $M$ has exactly one end which is non-parabolic. It is well-known that $\lambda_{1}(M)=1 / 4$. If $\Omega \subset \subset M$, then we can always find a domain $\Omega^{\prime}$ as in the statement of Proposition 4.1. Therefore $\alpha_{1}(M) \geq 1 / 4$. This shows that the assumption on the number of non-parabolic ends in the theorem cannot be improved without additional assumptions on the geometry.

We will need the following result of Li and Tam ([2], [3]).

THEOREM 4.3 (Li-Tam). Let $M$ be a complete, open Riemannian manifold with at least two disjoint non-parabolic ends. Denote one of them by $\mathcal{E}_{1}$. Let $p \in M$ and define $f_{R}$ by

$$
\begin{aligned}
\Delta f_{R}=0 & \text { in } B_{R}(p), \\
f_{R}=1 & \text { on } \partial B_{R}(p) \cap \mathcal{E}_{1}, \\
f_{R}=0 & \text { on } \partial B_{R}(p)-\mathcal{E}_{1} .
\end{aligned}
$$

Then there exists a sequence $R_{i} \rightarrow \infty$ such that $f_{R_{i}}$ converges uniformly on compact subsets of $M$ to a nonconstant, positive harmonic function with finite Dirichlet energy.

Proof of Theorem (4.2). Denote one of the nonparabolic ends by $\mathcal{E}_{1}$. Choose $p \in M$ and let $v_{R}$ denote the solution of

$$
\begin{aligned}
\Delta^{2} v_{R}=0 & \text { in } B_{R}, \\
\partial_{n} v_{R}=0 & \text { on } \partial B_{R}, \\
v_{R}=1 \quad \text { on } \partial B_{R} \cap \mathcal{E}_{1}, & v_{R}=0 \quad \text { on } \partial B_{R}-\mathcal{E}_{1} .
\end{aligned}
$$

As in the proof of Lemma 2.1, one can show

$$
\left\|\Delta v_{R}\right\|_{2}^{2}=\sup _{L_{H}^{2}\left(B_{R}\right)}\left(\oint_{\partial B_{R} \cap \mathcal{E}_{1}} * d h\right)^{2} / \int_{B_{R}} h^{2} .
$$

Also, as in the proof of Proposition 2.1, we have

$$
\int_{B_{R}} \zeta^{2}|\nabla h|^{2} \leq 4 \int_{B_{R}} h^{2}|\nabla \zeta|^{2}
$$

for all $h \in L_{H}^{2}\left(B_{R}\right), \zeta \in C_{c}^{\infty}\left(B_{R}\right)$. Choosing $\zeta$ to be a standard cut-off function with $\zeta \equiv 1$ on $B_{R / 2}$ gives

$$
\left(c / R^{2}\right) \int_{B_{R}} h^{2} \geq \int_{B_{R / 2}}|\nabla h|^{2}
$$

for all $h \in L_{H}^{2}\left(B_{R}\right)$, where $c$ is a constant independent of $R$. Thus by (15)

$$
\left\|\Delta v_{R}\right\|_{2}^{2} \leq\left(c / R^{2}\right) \sup _{L_{H}^{2}\left(B_{R}\right)}\left(\oint_{\partial B_{R} \cap \mathcal{E}_{1}} * d h\right)^{2} / \int_{B_{R / 2}}|\nabla h|^{2}=\left(c / R^{2}\right)\left(1 / \mu_{R / 2}\right),
$$


where $1 / \mu_{R / 2}$ is the harmonic capacity of $B_{R}-B_{R / 2}$. Let $f_{R_{i}}$ be as in Theorem 4.3. Note that by Dirichlet's Principle

$$
\int_{B_{R_{i}}}\left|\nabla v_{R_{i}}\right|^{2} \geq \int_{B_{R_{i}}}\left|\nabla f_{R_{i}}\right|^{2}
$$

Therefore for all $i$

$$
\alpha_{1}(M) \leq\left\|\Delta v_{R_{i}}\right\|_{2}^{2} /\left\|\nabla v_{R_{i}}\right\|_{2}^{2} \leq\left(c / R_{i}^{2}\right)\left(1 / \mu_{R_{i} / 2}\right) / \int_{B_{R_{1}}}\left|\nabla f_{R_{i}}\right|^{2} .
$$

Note that the sequence $1 / \mu_{R_{i} / 2}$ is decreasing with $i$ and by a well known property of harmonic functions

$$
\int_{B_{R_{1}}}\left|\nabla f_{R_{i}}\right|^{2} \rightarrow \int_{B_{R_{1}}}|\nabla f|^{2}>0
$$

The proof is completed by letting $i$ tend to $\infty$ in (18)

5. Stability of minimal Lagrangian submanifolds. Let $\left(X^{2 n}, \omega\right)$ be a symplectic manifold. An $n$-dimensional submanifold

$$
f: L \rightarrow X
$$

is called Lagrangian if $f^{*} \omega \equiv 0$.

We will be concerned with the special case when $X$ is a Kähler manifold, $\omega$ is its Kähler form and $L$ is a minimal Lagrangian submanifold. Let $J$ denote the almost complex structure of $X$ and note that $J$ defines an isometry between the tangent space and normal space at each point of $L$.

Let $\xi \in \Gamma_{c}(\perp L)$ (:= the space of compactly supported sections of the normal bundle of $L$ ) and let $\delta_{\xi}^{2}|L|$ denote the second variation of volume of $L$ in the direction $\xi$. Following $\mathrm{Oh}$ ([4]), we call L Hamiltonian stable if and only if

$$
\delta_{\xi}^{2}|L| \geq 0
$$

for all $\xi \in \Gamma_{c}(\perp L)$ of the form

$$
\xi=J(\nabla u), \quad u \in C^{\infty}(L) .
$$

In addition we will call $L$ weakly Hamiltonian stable if and only if (19) holds for all $\xi$ as in (20) with $u \in C_{c}^{\infty}(L)$.

Proposition 5.1. Let $X$ be a Kähler manifold and let $L$ be a minimal, Lagrangian submanifold of $X$. Assume that for some $c>0, \operatorname{Ric}_{X} \geq c \cdot d s_{X}^{2}$. Then the following hold.

(i) $L$ is Hamiltonian stable only if $\alpha_{1}(L) \geq c$.

(ii) $L$ is weakly Hamiltonian stable only if $\beta_{1}(L) \geq c$.

(iii) If $X$ is Einstein-Kähler with $\operatorname{Ric}_{X}=c \cdot d s_{X}^{2}, c>0$, then the conditions appearing in (i) and (ii) are both necessary and sufficient. 
PROOF. The second variation formula for minimal submanifolds shows that if $u$ is a smooth function such that $\nabla u$ has compact support then

$$
\delta_{J \nabla u}^{2}|L|=\int_{L}\left(|\nabla \nabla u|^{2}+\operatorname{Ric}_{L}(\nabla u, \nabla u)-\operatorname{Ric}_{X}(J \nabla u, J \nabla u)\right) .
$$

Integrating the Lichnerowicz formula

$$
\Delta \frac{1}{2}|\nabla u|^{2}=|\nabla \nabla u|^{2}+\operatorname{Ric}_{L}(\nabla u, \nabla u)+\langle\nabla u, \nabla \Delta u\rangle
$$

gives

$$
0=\int_{L}\left(|\nabla \nabla u|^{2}+\operatorname{Ric}_{L}(\nabla u, \nabla u)-(\Delta u)^{2}\right)
$$

so that

$$
\delta_{J \nabla u}^{2}|L| \leq \int_{L}\left((\Delta u)^{2}-c|\nabla u|^{2}\right)
$$

with equality if $X$ is Einstein with $c$ defined as in (iii). If $\nabla u$ has compact support in a domain $\Omega$, then clearly $u$ must be constant $c_{j}$ on each connected boundary component $\gamma_{j}$. Then

$$
w:=u-\sum c_{j} w_{j}
$$

is an element of $W_{0}^{2,2}(\Omega)$ and can hence be approximated by a smooth function with compact support. From this, (i), (ii) and (iii) follow.

As a consequence, we obtain.

THEOREM 5.1. Let $X$ be a Kähler manifold with $\operatorname{Ric}_{X} \geq c \cdot d s_{X}^{2}$ for some constant $c>0$ and let $L$ be a complete, open, minimal Lagrangian submanifold with at least two disjoint non-parabolic ends. Then L is not Hamiltonian stable.

EXAMPLES. In [1], Lawson constructed minimal immersions $F: \Sigma_{g} \rightarrow S^{3}$ of closed, genus $g$ surfaces. Let $G_{2,4}$ denote the Grassmannian of oriented planes in $\boldsymbol{R}^{4}$. The Gauss map $g: \Sigma_{g} \rightarrow G_{2,4}$ assigns to each point $p \in \Sigma$, the normal bundle to the immersion $F$ at the point $p$. Lawson refers to this map as the bipolar map and shows that $g$ is also defines a minimal immersion which induces the same conformal structure on $\Sigma$ as $F$. As noted in [5], this map also defines a Lagrangian surface in the Kähler-Einstein manifold $G_{2,4}$.

For $g>1$, we select a closed curve $\gamma \subset \Sigma$ which generates a nontrivial cyclic subgroup $\langle\gamma\rangle$ of $\pi_{1}(\Sigma)$. We identify $\pi_{1}(\Sigma)$ with the group of deck transformations of the universal cover $\tilde{\Sigma}$ and then consider the covering surface $\Sigma_{1}:=\tilde{\Sigma} /\langle\gamma\rangle$. The lift $g_{1}$ of $g$ to $\Sigma_{1}$ clearly defines a minimal, Lagrangian surface. It is not difficult to see that $\Sigma_{1}$, with the conformal structure induced from $g_{1}$, is conformally an annulus $\{1<|z|<R<\infty\}$. In particular, this means that $\Sigma_{1}$ has exactly two nonparabolic ends. Since $G_{2,4}$ has constant positive Ricci curvature, we can conclude from Theorem 5.1 that $g_{1}$ is not Hamiltonian stable. 
6. Appendix. In [7], Payne showed that for a convex domain in the plane, the inequality

$$
\beta_{1} \leq 4 \lambda_{1}
$$

holds. Here we will extend Payne's ideas to show the following.

THEOREM 6.1. Let $\Omega$ be a relatively compact subdomain with smooth, immersed boundary in a Riemannian manifold $M$. Suppose that $\partial \Omega$ has non positive mean curvature $H$ with respect to the outward pointing normal and that the Ricci curvature of $M$ satisfies

$$
\operatorname{Ric}_{M} \geq-\frac{3 a^{2}}{4} .
$$

Then

$$
\beta_{1}(\Omega) \leq 7 \lambda_{1}(\Omega)+3 a^{2}
$$

holds.

PROOF. Let $u$ be an eigenfunction for $\lambda_{1}$. Then

$$
\begin{aligned}
\left(\Delta u^{2}\right)^{2}=\left(2\left(|\nabla u|^{2}-\lambda_{1} u^{2}\right)\right)^{2} & =4\left(\lambda_{1}^{2} u^{4}+|\nabla u|^{4}+2 u \Delta u|\nabla u|^{2}\right), \\
\left|\nabla u^{2}\right|^{2} & =4 u^{2}|\nabla u|^{2} .
\end{aligned}
$$

It follows that

$$
\beta_{1} \leq \frac{\int 4\left(\lambda_{1}^{2} u^{4}+|\nabla u|^{4}+2 u \Delta u|\nabla u|^{2}\right) d \Sigma}{\int 4 u^{2}|\nabla u|^{2} d \Sigma} .
$$

Note that

$$
\begin{aligned}
0=\oint_{\partial \Omega} u^{3}\langle\nabla u, n\rangle d s & =\int\left(\left\langle\nabla u^{3}, \nabla u\right\rangle-\lambda_{1} u^{4}\right) \\
& =3 \int u^{2}|\nabla u|^{2}-\lambda_{1} \int u^{4} .
\end{aligned}
$$

Using this in (21), we obtain

$$
\beta_{1} \leq \lambda_{1}+\frac{\int|\nabla u|^{4}}{\int u^{2}|\nabla u|^{2}}=: \lambda_{1}+\theta .
$$

By Stoke's theorem, we have

$$
\begin{aligned}
\left(\int|\nabla u|^{4}\right)^{2} & =\left(-\int u \operatorname{div}\left(|\nabla u|^{2} \nabla u\right)\right)^{2} \\
& =\left(\int\left\langle u \nabla u, \nabla|\nabla u|^{2}-\lambda_{1} u \nabla u\right\rangle\right)^{2} \\
& \leq\left.\int u^{2}|\nabla u|^{2} \int|\nabla| \nabla u\right|^{2}-\left.\lambda_{1} u \nabla u\right|^{2} .
\end{aligned}
$$


This gives

$$
\begin{aligned}
\theta^{2} & \leq\left(\left.\int|\nabla| \nabla u\right|^{2}-\left.\lambda_{1} u \nabla u\right|^{2}\right) /\left(\int u^{2}|\nabla u|^{2}\right) \\
& =\lambda_{1}^{2}+\left.\left.\int|\nabla| \nabla u\right|^{2}\right|^{2} / \int u^{2}|\nabla u|^{2}-2 \lambda_{1} \int\left\langle u \nabla u, \nabla|\nabla u|^{2}\right\rangle / \int u^{2}|\nabla u|^{2} .
\end{aligned}
$$

We expand the numerator in the last term

$$
\begin{aligned}
\int\left\langle u \nabla u, \nabla|\nabla u|^{2}\right\rangle & =(1 / 2) \int\left\langle\nabla u^{2}, \nabla|\nabla u|^{2}\right\rangle \\
& =(-1 / 2) \int|\nabla u|^{2} \Delta u^{2} \\
& =-\int\left(|\nabla u|^{4}-\lambda_{1} u^{2}|\nabla u|^{2}\right) .
\end{aligned}
$$

Inserting this in the estimate for $\theta^{2}$ and replacing in (22), gives

$$
\theta^{2} \leq\left(\left.\left.\int|\nabla| \nabla u\right|^{2}\right|^{2}\right) /\left(\int u^{2}|\nabla u|^{2}\right)+2 \lambda_{1} \theta-\lambda_{1}^{2}=: \Gamma+2 \lambda_{1} \theta-\lambda_{1}^{2} .
$$

We may assume that $u>0$ holds in $\Omega$ so that the outward pointing normal to $\partial \Omega$ is $n=$ $-\nabla u /|\nabla u|$. Thus,

$$
\begin{aligned}
\partial_{n}|\nabla u|^{2} & =2\left\langle\nabla_{n} \nabla u, \nabla u\right\rangle=-2|\nabla u|\left\langle\nabla_{n} \nabla u, n\right\rangle \\
& =2|\nabla u|\left\langle\nabla_{e_{i}} \nabla u, e_{i}\right\rangle=-2|\nabla u|^{2}\left\langle\nabla_{e_{i}} n, e_{i}\right\rangle \\
& =2 n H|\nabla u|^{2},
\end{aligned}
$$

where $n=\operatorname{dim} M$. Using the assumption on $H$ and the Lichnerowicz formula, we obtain

$$
\begin{aligned}
0 & \geq 2 \oint_{\partial \Omega} 2 n H|\nabla u|^{4}=\oint_{\partial \Omega}|\nabla u|^{2} \partial_{n}|\nabla u|^{2} \\
& =\left.\left.\int|\nabla| \nabla u\right|^{2}\right|^{2}+\int 2|\nabla u|^{2}\left(\left|\nabla^{2} u\right|^{2}+\operatorname{Ric}(\nabla u, \nabla u)-\lambda_{1}|\nabla u|^{2}\right) .
\end{aligned}
$$

Using $\left.\left.|\nabla| \nabla u\right|^{2}\right|^{2} \leq 4|\nabla u|^{2}\left|\nabla^{2} u\right|^{2}$ and the previous inequality, we have

$$
\begin{aligned}
0 & \geq 2 \lambda_{1} \int|\nabla u|^{4}-2 \int|\nabla u|^{2} \operatorname{Ric}(\nabla u, \nabla u) \\
& \geq \int\left(2|\nabla u|^{2}\left|\nabla^{2} u\right|^{2}+\left.\left.|\nabla| \nabla u\right|^{2}\right|^{2}\right) \\
& \geq\left.\left. 3 \int|\nabla| \nabla u\right|^{2}\right|^{2} .
\end{aligned}
$$

We arrive at

$$
\Gamma \leq\left((4 / 3) \int\left(\lambda_{1}|\nabla u|^{4}-|\nabla u|^{2} \operatorname{Ric}(\nabla u, \nabla u)\right) / \int u^{2}|\nabla u|^{2} .\right.
$$

This gives

$$
\theta^{2} \leq(10 / 3) \lambda \theta-\lambda^{2}+a^{2} \theta
$$


from which the result easily follows.

\section{REFERENCES}

[ 1 ] H. B. Lawson, Complete minimal surfaces in $S^{3}$, Ann. of Math. (2) 92 (1970), 335-374.

[2 $]$ P. Li AND L. F. TAM, Harmonic functions and the structure of complete manifolds, J. Differential Geom. 35 (1992), 359-383.

[ 3 ] P. Li AND L. F. TAM, Positive harmonic functions on complete manifolds with non-negative curvature outside a compact set, Ann. of Math. (2) 125 (1987), 171-207.

[4] Y. G. OH, Second variation and stability of minimal Lagrangian submanifolds, Invent. Math. 101 (1990), 501-519.

[ 5 ] B. PALmer, Buckling eigenvalues, Gauss maps and Lagrangian submanifolds, Differential Geom. Appl. 4 (1994), 391-403.

[ 6 ] B. PAlmer, Hamiltonian minimality and Hamiltonian stability of Gauss maps, Differential Geom. Appl. 7 (1997), 51-58.

[ 7 ] L. E. PAYne, A Note on inequalities for plate eigenvalues, J. Math. and Phys. 39 (1960/1961), 155-159.

DEPARTMENT OF MATHEMATics

IDAHO STATE UNIVERSITY

POCATELLO, IDAHO

83209

U.S.A.

E-mail address: palmbenn@isu.edu 